\title{
EINSTEIN GRAVITY FROM RESTRICTED COORDINATE INVARIANCE
}

\author{
W. BUCHMÜLLER ${ }^{\mathrm{a}, \mathrm{b}}$ and N. DRAGON ${ }^{\mathrm{a}, \mathrm{c}}$ \\ a Institut für Theoretische Physik, Universität Hannover, D-3000 Hannover, Fed. Rep. Germany \\ b Deutsches Elektronen-Synchrotron DESY, D-2000 Hamburg 52, Fed. Rep. Germany \\ c Institut für Theoretische Physik, Universität Karlsruhe, D-7500 Karlsruhe, Fed. Rep. Germany
}

Received 16 March 1988

\begin{abstract}
We show that Einstein's equations for the gravitational field can be derived from an action which is invariant only under restricted coordinate transformations which preserve the volume. The only difference compared to a general covariant theory concerns the cosmological constant, which becomes an arbitrary initial condition.
\end{abstract}

Einstein's equations describe successfully all known gravitational phenomena. They can be derived from an action which is invariant under general coordinate transformations, and the Principle of General Covariance is the generally accepted basis of field theories which include gravity [1].

In this note we want to point out that already a smaller symmetry is sufficient to obtain Einstein's equations. It suffices to require invariance under restricted coordinate transformations which preserve the volume. We will show that theories with invariance under restricted coordinate transformations are classically equivalent to theorics with invariance under general coordinate transformations. The only difference concerns a constant, the cosmological term. In general covariant theories the cosmological constant is a parameter of the lagrangian, whereas in restricted covariant theories it appears as an arbitrary initial condition.

Let us consider an infinitesimal general coordinate transformation

$x^{\prime \mu}=x^{\mu}-\epsilon^{\mu}(x)$.

The metric tensor $g_{\mu \nu}$ changes under this local translation by its Lie derivative,

$$
\begin{aligned}
& \delta g_{\mu \nu}(x)=g_{\mu \nu}^{\prime}(x)-g_{\mu \nu}(x) \\
& \quad=\epsilon^{\lambda} \partial_{\lambda} g_{\mu \nu}(x)+\partial_{\mu} \epsilon^{\lambda}(x) g_{\lambda \nu}(x) \\
& \quad+\partial_{\nu} \epsilon^{\lambda}(x) g_{\mu \lambda}(x),
\end{aligned}
$$

which yields for the volume element $\sqrt{g}=$ $\left[-\operatorname{det}\left(g_{\mu \nu}\right)\right]^{1 / 2}$

$\delta \sqrt{g}=\partial_{\lambda}\left(\epsilon^{\lambda} \sqrt{g}\right)$.

We define restricted coordinate transformations as volume preserving transformations, which means that $\sqrt{g}$ transforms as a scalar field:

$\delta \sqrt{g}=\epsilon^{\lambda} \partial_{\lambda} \sqrt{g}$.

From eqs. (3) and (4) we conclude that restricted coordinate transformations are local translations with vanishing divergence

$x^{\prime \mu}=x^{\mu}-\epsilon^{\mu}(x), \quad \partial_{\mu} \epsilon^{\mu}(x)=0$.

For restricted coordinate transformations we may rewrite the metric as

$g_{\mu \nu}=\mathrm{e}^{2 \sigma} \bar{g}_{\mu \nu}, \quad \operatorname{det}\left(\bar{g}_{\mu \nu}\right)=-1$,

where $\sigma$ is an ordinary scalar field.

In order to obtain local actions which are invariant under restricted coordinate transformations (5) one constructs tensors out of the fields and their partial derivatives. Partial derivatives of tensors can be completed to tensors by means of the Christoffel symbols

$\bar{\Gamma}_{\mu \nu}{ }^{\rho}=\frac{1}{2} g^{\rho \sigma}\left(\partial_{\mu} g_{\sigma \nu}+\partial_{\nu} g_{\mu \sigma}-\partial_{\sigma} g_{\mu \nu}\right)$,

which are in one-to-one correspondence with the firstorder partial derivatives of $\bar{g}_{\mu \nu}$. Hence all tensors 
which can be constructed from $\bar{g}_{\mu \nu}$ and its derivatives can also be obtained from $\bar{g}_{\mu \nu}, \bar{\Gamma}_{\mu \nu}{ }^{\rho}$ and derivatives of $\bar{\Gamma}_{\mu \nu}{ }^{\rho}$. The Christoffel symbols do not transform as a tensor,

$$
\begin{aligned}
& \bar{\Gamma}_{\mu \nu}{ }_{\mu \nu}{ }^{\rho}=\bar{\Gamma}_{\mu \nu}{ }^{\rho} \\
& \quad+\left(\epsilon^{\lambda} \partial_{\lambda} \bar{\Gamma}_{\mu \nu}{ }^{\rho}+\partial_{\mu} \epsilon^{\lambda} \bar{\Gamma}_{\lambda \nu}{ }^{\rho}+\partial_{\nu} \epsilon^{\lambda} \bar{\Gamma}_{\mu \nu}{ }^{\rho}-\partial_{\lambda} \epsilon^{\rho} \bar{\Gamma}_{\mu \nu}{ }^{\lambda}\right) \\
& \quad+\partial_{\mu} \partial_{\nu} \epsilon^{\rho},
\end{aligned}
$$

due to the last term in (8). In the case of restricted gauge transformations ( 5 ) this term does not contributc to $\delta_{\rho}^{\mu} \bar{\Gamma}_{\mu \nu}^{\rho}$. However, this tensor vanishcs identically,

$$
\bar{\Gamma}_{\mu \nu}{ }^{\mu}=0 \text {, }
$$

because of (7) and $\bar{\Gamma}_{\mu \nu}{ }^{\mu}=\partial_{\nu} \ln \sqrt{\bar{g}}$.

The antisymmetric combination of first-order partial derivatives of Christoffel symbols, $\partial_{\mu} \bar{\Gamma}_{\nu \lambda}{ }^{p}-$ $\partial_{\nu} \bar{\Gamma}_{\mu \lambda}{ }^{p}$, can be completed to the Riemann tensor $\bar{R}_{\mu \nu \lambda}{ }^{p}$. The symmetric combination cannot be completed to a tensor because of the last term in (8). This term does not contribute to

$\delta_{\rho}^{\mu}\left(\partial_{\mu} \bar{\Gamma}_{\nu \lambda}{ }^{\rho}+\partial_{\nu} \bar{\Gamma}_{\mu \lambda}{ }^{\rho}\right)=\delta_{\rho}^{\mu}\left(\partial_{\mu} \bar{\Gamma}_{\nu \lambda}{ }^{\rho}-\partial_{\nu} \bar{\Gamma}_{\mu \lambda}{ }^{\rho}\right)$,

where we have used eq. (9). However, the expression (10) can be completed to the Ricci tensor $\bar{R}_{\mu \nu}$ and does not yield a new tensor. This reasoning is easily extended to higher partial derivatives of $\bar{\Gamma}_{\mu \nu}{ }^{p}$. We conclude that the tensors under restricted coordinate transformations, which one can construct from $\bar{g}_{\mu \nu}$ and its partial derivatives, are identical to the tensors under general coordinate transformations, i.e., there are only $g_{\mu \nu}, \bar{R}_{\mu \nu \rho \sigma}$ and its covariant derivatives.

Hence the most general lagrangian which can be constructed from $\bar{g}_{\mu \nu}$ and additional matter fields $\Phi$, and which yields second order differential equations for $\bar{g}_{\mu \nu}$, is given by

$L=\frac{1}{2} \chi^{2}(\Phi) \bar{R}(\bar{g})+\bar{L}(\bar{g}, \Phi)$.

Here $\chi^{2}$ is a scalar field depending on the matter fields $\Phi$ (which include the field $\sigma$ defined in (6)) with positive vacuum expectation valuc,

$$
\left\langle\chi^{2}(\Phi)\right\rangle_{0}>0
$$

$\bar{R}$ is the Ricci scalar and $\bar{L}$ is a scalar constructed from $\bar{g}_{\mu \nu}, \bar{\Gamma}_{\mu \nu}{ }^{\rho}$ and the matter fields $\Phi$. Compared to lagrangians resulting from the stronger requirement of general coordinate invariance the lagrangian (11) does not contain the volume factor $\sqrt{\bar{g}}$ which was fixed to 1 in eq. (6). Instead it contains an additional scalar field $\sigma$ (cf. eq. (6)) which is counted as matter field.

It is convenient to rewrite the lagrangian (11) in terms of the Weyl-rescaled metric

$g_{\mu \nu}=\chi^{2}(\Phi) \bar{g}_{\mu \nu}$.

With $\bar{L}(\bar{g}, \Phi)=\chi^{4} L(g, \Phi)$ one obtains

$L=\sqrt{g}\left[\frac{1}{2} R(g)+6 g^{\mu \nu} \chi^{-2} \partial_{\mu} \chi \partial_{\nu} \chi+L(g, \Phi)\right]$.

In this form the invariance under general coordinate transformations is broken through the constraint

$\sqrt{g}=\chi^{4}(\Phi)$,

which corresponds to a partial gauge fixing [2]. This constraint can be incorporated by means of a Lagrange multiplier field $A(x)$ and one finally obtains

$L=\sqrt{g}\left[\frac{1}{2} R(g)+L_{\mathrm{M}}(g, \Phi)-\chi^{-4}(\Phi) A\right]+A$,

where $L_{\mathrm{M}}$ is the sum of all terms containing matter fields $\Phi$. In (16) the general coordinate invariance is broken only through the last term.

The equations of motion which one obtains from the lagrangian (16) for the matter fields $\Phi$ depend on the Lagrange multiplier field $A$. Varying $A$ gives the constraint (15), variation with respect to $g_{u v}$ yields Einstein's equations:

$R_{\mu \nu}-\frac{1}{2} g_{\mu \nu} R=-T_{\mu \nu}$,

where

$T_{\mu \nu}=\frac{2}{\sqrt{g}} \frac{\delta S_{\mathrm{M}}}{\delta g^{\mu \nu}}$,

$S_{\mathrm{M}}=\int \mathrm{d}^{4} x\left\{\sqrt{g}\left[L_{\mathrm{M}}(g, \Phi)-\chi^{-4}(\Phi) A\right]+\Lambda\right\}$.

There is, however, a subtle difference. If the action (16) were invariant under general coordinate transformations the energy momentum tensor $T_{\mu \nu}$ would be covariantly conserved,

$\mathrm{D}^{\mu} T_{\mu \nu}=0$

for arbitrary gravitational fields $g_{\mu \nu}$ and for matter fields $\Phi$ satisfying the equations of motion. In the case of restricted coordinate invariance equation (20) follows only from Einstein's equations (17). This 
does not overdetermine the matter fields, yet it fixes precisely the Lagrange multiplier field $A$.

To see this, consider the variation of the matter part of the action under an infinitesimal general coordinate transformation:

$$
\begin{aligned}
& \delta S_{\mathrm{M}}=\int \mathrm{d}^{4} x \epsilon^{\mu} \partial_{\mu} A \\
& \quad=\int \mathrm{d}^{4} x\left(\frac{\delta S_{\mathrm{M}}}{\delta \Phi} \delta \Phi+\frac{\delta S_{\mathrm{M}}}{\delta \Lambda} \delta A+\frac{1}{2} \sqrt{g} T_{\mu \nu} \delta g^{\mu \nu}\right) .
\end{aligned}
$$

For fields $\Phi$ and $A$ satisfying the equations of motion, and for gauge variations

$\delta g^{\mu \prime \prime}=-\mathrm{D}^{\mu} \epsilon^{\prime \prime}-\mathrm{D}^{\prime \prime} \epsilon^{\mu}$

one obtains after integration by parts

$0=\int \mathrm{d}^{4} x \epsilon^{\mu}\left(\partial_{\mu} \Lambda-\sqrt{g} \mathrm{D}^{\nu} T_{\nu \mu}\right)$,

which implies for arbitrary $\epsilon^{\mu}(x)$

$\sqrt{g} \mathrm{D}^{\nu} T_{\nu \mu}=\partial_{\mu} \Lambda$.

From eqs. (20) and (24) one obtains

$A=$ const .

The value of this constant depends on the initial conditions and is not a parameter of the lagrangian. In the special case $\chi^{2}(\Phi)=1$ the lagrange multiplier field $A$ adds a covariantly constant term to the energy momentum tensor, which corresponds to the contribution of a cosmological constant. For general $\chi^{2}(\Phi)$ the choice of the constant $A$ similarly determines the curvature of the ground state. There the matter fields are constant and again the ground state curvature becomes an arbitrary initial condition.
We conclude that theories with invariance under general coordinate transformations are classically equivalent to theories with invariance under restricted coordinate transformations. The corresponding equations of motion are identical except for the cosmological term. In general covariant theories the cosmological constant is a parameter of the lagrangian, in restricted covariant theories it appears as an initial condition.

We would like to thank M. Kreuzer for enjoyable discussions.

Note added. After this paper was submitted we became aware of the work of van der Bij, van Dam and $\mathrm{Ng}$ [3] who previously considered a theory of gravity with invariance under restricted coordinate transformations only. Our result concerning the role of the cosmological constant is already contained in this paper. This result was also obtained by Wilczek and Zee [4] who investigated various aspects of the cosmological constant problem in theories where the invariance under coordinate transformations is restricted to volume prescrving transformations.

\section{References}

[1] See, e.g. S. Weinberg, Gravitation and cosmology (New York, $1972)$.

[2] W. Buchmüller and N. Dragon, Gauge fixing and the cosmological constant, preprint ITP-UH 1/88, DESY 88-019 (1988).

[3] J.J, van der Bij, H. van Dam and Y.J. Ng, Physica A 116 (1982) 307.

[4] A. Zee, in: High-energy physics, eds. B. Kursunoglu et al. (Plenum, New York, 1985). 